\title{
Focusing a Gaussian beam in nanostructured non-periodic GRIN microlenses
}

\author{
Jedrzej M. Nowosielski, ${ }^{* 1,2}$ Ryszard Buczynski, ${ }^{1,2}$, Florian Hudelist, ${ }^{1}$ Andrew Waddie, ${ }^{1}$ Dariusz Pysz, ${ }^{3}$ \\ Ryszard Stępien, ${ }^{3}$ Ireneusz Kujawa, ${ }^{3}$ and Mohammad R. Taghizadeh ${ }^{1}$ \\ ${ }^{I}$ School of Engineering and Physical Sciences, Heriot-Watt University, Edinburgh EH14 4AS, UK \\ ${ }^{2}$ University of Warsaw, Department of Physics, 7 Pasteur, 02-093 Warsaw, Poland \\ ${ }^{3}$ Institute of Electronic Materials Technology, 133 Wolczynska, 01-919 Warsaw, Poland
}

Received March 19, 2010; accepted March 25, 2010; published March 31, 2010

\begin{abstract}
Modeling the propagation of a Gaussian beam in a nanostructured gradient index microlens is reported. The nanostructured GRIN microlens is composed of subwavelength discrete dielectric rods. We compare the performance of nanostructured gradient index microlenses against conventional GRIN microlenses for Gaussian beam focusing. The 3D FDTD method is used for numerical analysis of optical systems.
\end{abstract}

Gradient-Index (GRIN) rod microlenses are very attractive optical components for compact, high level optical systems [1]. Flat input and output surfaces make them easy to integrate with other optical components. They have good imagining properties with a high numerical aperture. Their performance is limited due to the low index gradient and limited control of refractive index distribution. Moreover, it is difficult and expensive to fabricate 1D and 2D arrays of GRIN rod microlenses using standard fabrication methods [2].

Recently, we have introduced a new type of nanostructured Gradient Index (nGRIN) microlens [3] fabricated by using the standard stack and draw technique widely used for photonic crystal fibers development. We have also shown the possibility of elliptical nGRIN fabrication with this technique [4].

Nanostructured microlenses consist of many subwavelength diameter nanorods. These rods are parallel to each other and subwavelength in diameter. There are two types of rods, each made of a different type of glass with a different refractive index. The rods are stacked according to an arbitrary pattern to create a preform. Next the preform is scaled down using a fiber drawing tower. Afterwards, further scaling down processes are repeated in order to obtain the final lens structure of diameter $10-$ $200 \mu \mathrm{m}$. A single glass rod in the nGRIN lens has a feature size of the order of magnitude of $100 \mathrm{~nm}$. Finally, the fabricated lens rod is cut into slices which are typically a few hundred microns thick.

The nGRIN lens principle of operation is based on the effective medium theory. The subwavelength diameter of rods results in the fact that some continuous distribution of effective (averaged) refractive index is created within the structure. The concept of nanostructured microlenses and the fabrication procedure is presented in detail in [3].

With this new technique it is possible to obtain a very high gradient of effective refractive index up to $\Delta \mathrm{n}=0.1$ per $5 \mu \mathrm{m}$ with respect to standard GRIN lenses $(\Delta \mathrm{n} \sim 0.1$ per $250 \mu \mathrm{m}$ ). A very high f-number (up to 1 ), as well as $2 \mathrm{D}$ arrays with the filling factor close to $100 \%$ can be achieved with this method. In previous papers we have shown the performance of nGRIN lenses under plane wave illumination $[3,4]$. In this paper we investigate numerically Gaussian beam propagation through the lenses.

The refractive index distribution within the GRIN lens is given by [5]

$$
\begin{gathered}
n(x, y)=n_{\max }\left[1-\frac{g_{0}^{2}}{2}\left(x^{2}+y^{2}\right)\right], \\
g_{0}^{2}=\frac{8\left(n_{0}-n_{\text {min }}\right)}{n_{0} d^{2}}
\end{gathered}
$$

where $n_{\max }$ denotes the refractive index in the centre of the lens, $g_{0}$ - gradient parameter, $d$-diameter of the lens, and $n_{\min }$ is the refractive index at the edge of the lens.

The behavior of a Gaussian beam in the gradient index media depends on the relation between the Gaussian beam waist $w_{o}$ and a half-width of the fundamental mode of gradient index medium $w_{f m}$. The half-width diameter of a Gaussian beam that propagates along the $\mathrm{z}$ axis is given by

$$
w^{2}(z)=w_{0}^{2}\left[\cos ^{2}\left(g_{0} z\right)+\frac{w_{f m}^{4}}{w_{0}^{4}} \sin ^{2}\left(g_{0} z\right)\right],
$$

where

$$
w_{f m}=\left(\frac{\lambda_{0}}{n_{\max } g_{0} \pi}\right)^{1 / 2}
$$

*E-mail: jmn8@hw.ac.uk 
If $w_{o}=w_{f m}$ then $w(z)$ is independent of $\mathrm{z}$ and the Gaussian beam will propagate in the medium without oscilations.. If $w_{o}>w_{f m}$ then the Gaussian beam is focused within the gradient medium: $w(z)$ decreases at the distance $0<z<\pi /\left(2 g_{0}\right)$ and reaches the minimum for $z=\pi /\left(2 g_{0}\right)$. If $w_{o}<w_{f m}$ then the Gaussian beam expands within the gradient medium: $w(z)$ increases at the distance $0<z<\pi /\left(2 g_{0}\right)$ and reaches the maximum for $z=\pi /\left(2 g_{0}\right)$. The GRIN rod lens with a length of $\pi /\left(2 g_{0}\right)$ is called a quarter pitch lens.

To study the focusing properties of an nGRIN lens we assume that $w_{o}>w_{f m}$ and the length of the gradient index medium is equal to that of a quarter pitch lens. We have designed a continuous GRIN lens with refractive index distribution characterized by gradient parameter $g_{0}=45$ $\mathrm{mm}^{-1}$, diameter $d=10 \mu \mathrm{m}$ and refractive indexes $n_{\max }=1.619$ and $n_{\min }=1.578$. The refractive index distribution within a continuous GRIN microlens is described by (1). In this case the quarter pitch value is $34.9 \mu \mathrm{m}$.

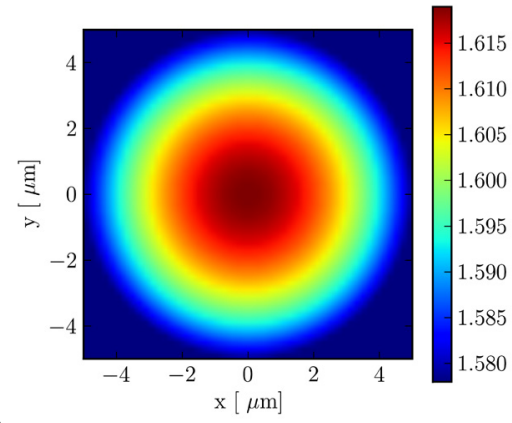

a)

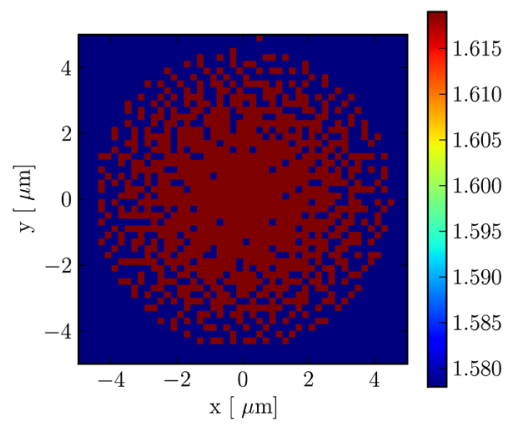

b)

Fig.1. Cross-section of refractive index distribution in a continuous GRIN microlens (a) and corresponding nanostructured GRIN microlenses (b).

The nGRIN lens design was based on the lens above, using the algorithm described in [3]. In this case, it consists of 2500 nanorods ordered in a square lattice with $200 \times 200 \mathrm{~nm}$ diameter. It is composed of two types of nanorods only with refractive indexes 1.619 and 1.578 .
According to 3D FDTD simulations with plane wave illumination, both lenses have similar effective properties such as quarter pitch and focal spot profile and diameter. The refractive index distribution in a cross-section of the continuous GRIN microlens and the corresponding nGRIN microlens are presented in Fig.1.

3D simulations are performed with the FDTD method using the MEEP software package [6]. Both the continuous and nanostructured GRIN microlens are illuminated with a planar Gaussian beam polarized linearly along the $Y$ axis with a beam half-width of $w_{o}=5$ $\mu \mathrm{m}$. A vacuum wavelength $\lambda_{0}=1 \mu \mathrm{m}$ is assumed. The size of the computational cell is $18 \mu \mathrm{m} \times 18 \mu \mathrm{m} \times 42 \mu \mathrm{m}$. Spatial step $\Delta x$ is set to $\lambda_{0} / 20=50 \mathrm{~nm}$. Time step $\Delta t$ is set to ensure numerical stability.

The Z-component of the Poynting vector in longitudinal section of the lens and beam profile at the quarter pitch lens is calculated for the continuous and the nGRIN microlenses. We also compare the output beam half-width obtained by FDTD simulation the with prediction of the scalar GRIN theory given by (3). The intensity distribution within the continuous GRIN microlens is presented in Fig. 2 and the corresponding intensity distribution within the nGRIN microlens is shown in Fig. 3. Intensity is represented by the $Z$ component $S_{z}$ of the averaged in a time Poynting vector.

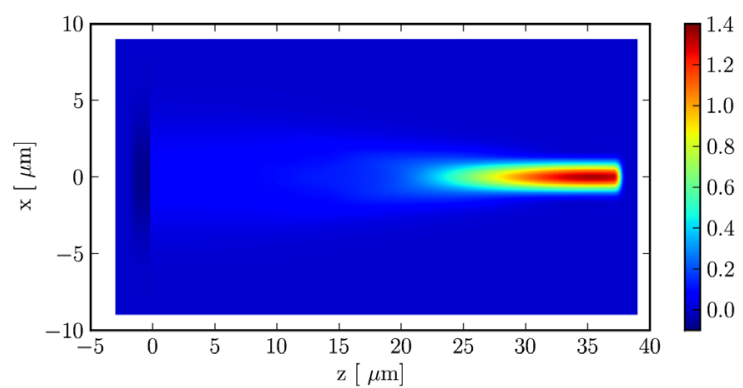

Fig.2. Intensity distribution for the continuous GRIN microlens -a crosssection in the XZ plane of 3D FDTD simulation.. The Gaussian beam propagates on-axis along the $\mathrm{Z}$ axis.

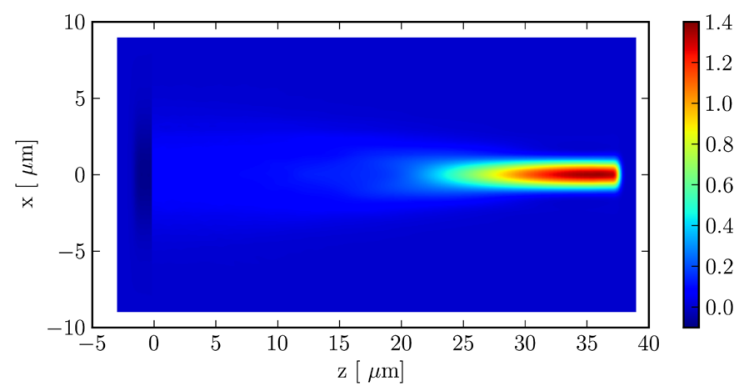

Fig.3. Intensity distribution for the nanostructured GRIN microlens - a cross-section in the XZ plane of 3D FDTD simulation.. The Gaussian beam propagates on-axis along the $\mathrm{Z}$ axis. 
The presented intensity distributions show similar performance in the case of a continuous and a nanostructured GRIN microlens. The quarter pitch obtained from the FDTD simulations in the case of continuous and nanostructured GRINs are $35 \mu \mathrm{m}$ and is very similar to the one predicted during the design of the lenses. A cross-section at the quarter pitch plane is presented in Fig. 4 and Fig. 5 for continuous and nanostructured GRIN lenses, respectively.

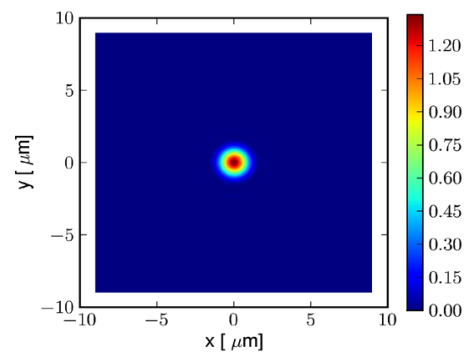

Fig.4. Intensity distribution of a focused Gaussian beam at the quarter pitch plane for the continuous GRIN microlens -a cross-section in the $\mathrm{XY}$ plane of 3D FDTD simulation.

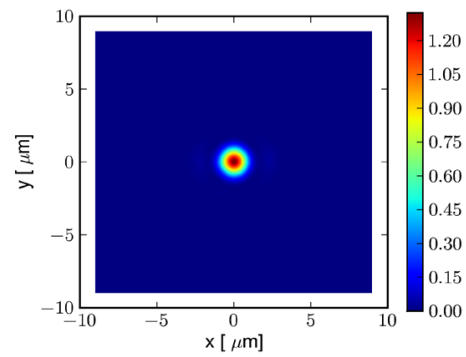

Fig.5. Intensity distribution of a focused Gaussian beam at the quarter pitch plane for the nanostructured GRIN microlens -a cross-section in the XY plane of 3D FDTD simulation

For both types of lenses we have obtained similar intensity distributions, as well as diameters of the focused beams. For more accurate analysis of the Gaussian properties of a focused beam, 1D profiles of continuous and nanostructured GRIN microlenses are compared with each other. The 1D beam profiles for continuous and nanostructured lenses are shown in Fig. 6. In addition, we compare the obtained results with the Gaussian beam profile predicted by the GRIN paraxial theory given by [5] using formula (3).

The profiles obtained for both lenses have the same shape. We have fitted the Gaussian profile to the results obtained by the FDTD simulation and a perfect match is obtained (Fig.6). The half-width of the beam at the quarter pitch plane is $1.15 \mu \mathrm{m}$ for both the continuous GRIN microlens and the nGRIN microlens. The theoretical value of the half-width calculated according to formula (3) is smaller and it is equal to $0.87 \mu \mathrm{m}$. The difference between theoretical and simulated results is not related to the nanostructured nature of the lens, since the continuous lens shows the same difference. Most probably, the difference is a result of the high gradient in both lenses and both equation (3) as well as the paraxial gradient index theory may not be valid for such large gradients with respect to standard GRIN microlenses gradients of a level of $1 \mathrm{~mm}^{-1}$. Further study of this problem is needed.

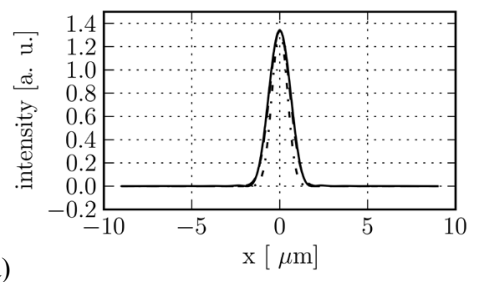

a)

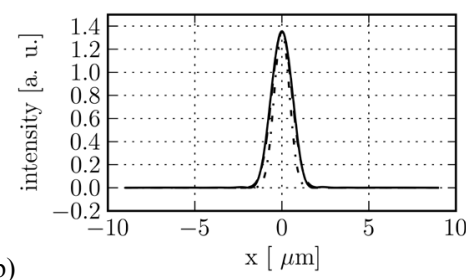

Fig.6. The profiles of a focused Gaussian beam at the quarter pitch plane for a continuous (a) and the nanostructured (b) GRIN lens. The solid line presents the results obtained with FDTD simulations, while the dash line presents a Gaussian beam fit for the simulated profile. A dash-dot line represents a calculated profile based on eq. (3) given by the scalar paraxial theory .

In this paper we have shown equivalence between the continuous GRIN and nanostructured GRIN microlenses for Gaussian beam propagation. In both cases the effective parameters are the same and the focused beam has a Gaussian profile. It was also shown that the standard paraxial GRIN theory yields close but not similar results and cannot be applied with full confidence for high contrast GRIN microlenses.

\section{References}

[1] W. J. Tomlinson, "Application of GRIN rod lenses in optical fiber communication systems," Appl. Opt. 19, 1127-1138 (1980).

[2] S. Sinzinger, and J. Jahns, Microoptics Wiley-VCH, Weinheim, 2003.

[3] F. Hudelist, R. Buczynski, A. J. Waddie, and M. R. Taghizadeh, "Design and fabrication of nano-structured gradient index microlenses," Opt. Express 17, 3255-3263 (2009).

[4] F. Hudelist, J. M. Nowosielski, R. Buczynski, A. J. Waddie, and M. R. Taghizadeh, "Nanostructured elliptical gradient-index microlenses," Opt. Lett. 35, 130-132 (2010).

[5] C. Gomez-Reino, M. V. Perez, and C. Bao, Gradient-Index Optics: Fundamentals and Applications Springer, Berlin, 2002

[6] A. Farjadpour, D. Roundy, A. Rodriguez, M. Ibanescu, P. Bermel J. D. Joannopoulos, S. G. Johnson, and G. Burr, "Improving accuracy by subpixel smoothing in FDTD," Opt. Lett. 31 (20), 297 (2006). 